\title{
DESENVOLVIMENTO DE TECIDOS DE SISAL PARA UTILIZAÇÃO EM
} COMPÓSITOS POLIMÉRICOS

\section{Camila Cruz da Silva}

Bolsista PIBIC - IFRN - Campus Natal Central. E-mail: camilacdasilva@hotmail.com

Ricardo Alex Dantas da Cunha

Bolsista CNPq - PIBITI - IFRN - Campus Natal Central.

E-mail: ricardoalexdcunha@hotmail.com

Renata Carla Tavares dos Santos Felipe

Professora do IFRN - Campus Natal Central. E-mail: rcfelipe@cefetrn.br

\author{
Raimundo Nonato Barbosa Felipe
}

Professor do IFRN - Campus Natal Central. E-mail: nonatofelipe@cefetrn.br

\section{RESUMO}

A utilização das fibras naturais (lignocelulosicas) como reforço, em materiais compósitos polimérico, é algo que vem crescendo significativamente dia, após dia. Devido ao fato desses materiais serem biodegradáveis, provém de fontes renováveis, desenvolvendo assim as regiões onde são extraídas possibilitando dessa forma a permanência do homem no campo, fazendo parte assim de um modelo ecologicamente correto. Dentre essas fibras dá um destaque a fibra de sisal,onde esta está sendo bastante estudada e utilizada na indústria automobilística. A fibra é extraída da folha do agave sisalina, e que foi introduzida no Brasil em meados de 1900, tendo como maiores produtores os estados da Bahia, Paraíba e Rio Grande do Norte. O objetivo deste trabalho é o desenvolvimento de dois tecidos de sisal para a utilização em materiais compósitos poliméricos, já que estes não são encontrados na indústria. Dessa forma foram fabricados dois tecidos sendo um do tipo plano e o outro do tipo plano basket, onde estes foram obtidos em teares manuais.

PALAVRAS-CHAVE: Materiais Compósitos, Sisal e Tecidos. 


\section{DESENVOLVIMENTO DE TECIDOS DE SISAL PARA UTILIZAÇÃO EM COMPÓSITOS POLIMÉRICOS}

\section{INTRODUÇÃO}

Um material composto pode ser definido como um material formado por dois ou mais materiais, com características e aspectos diferentes, no entanto quando analisados macroscopicamente o mesmo é homogêneo podendo possuir fibras longas ou curtas (Gay 1991). Nesses materiais pode-se destacar que são formados por uma fase de reforço, que podem ter a forma de filamento; e uma outra fase, conhecida como matriz que tem a função aglutinante, que faz com que o reforço trabalhe de forma integrada suportando os esforços mecânicos (Tabela 1) (Pardini et.all, 2006). São inúmeras as aplicações desses materiais, onde dos quais podemos citar a indústria automobilística (fabricação de pará choque, corroeria de caminhão), aeronáutica (hélice de helicóptero), náutica (fabricação de barco) química (taques para armazenamento de produtos químicos) entre outras indústrias, (Albuquerque, 1999).

Hoje em dia a utilização desses materiais na indústria é algo que vem crescendo gradativamente, dia após dia, e com esse crescimento a preocupação em se substituir o material sintético pelo natural. As fibras naturais têm sido estudadas ao longo de décadas, no que se referem a sua constituição, propriedades mecânicas e seus possíveis usos. As vantagens do uso das fibras naturais sobre as fibras sintéticas são: biodegradáveis, baixo custo, baixa densidade, boa tenacidade, boas propriedades térmicas e uso reduzido de instrumentos para o seu tratamento ou processamento (BARROS). A utilização dessas fibras, como por exemplo, as fibras de abacaxi, bananeira, coco, carauá, juta, piaçava, rami, sisal no lugar das fibras sintéticas como, por exemplo; fibras de vidros e carbono.

Dentre as fibras naturais a fibra de sisal se mostra promissora na utilização de materiais compósitos devido as suas propriedades e a sua boa disponibilização no mercado. A fibra de sisal é extraída da folha do agave sisalina, planta esta originaria do México, e que foi introduzida no Brasil por volta de 1900 na região nordeste, onde hoje em dia tem como seus maiores produtores os estados da Bahia, Paraíba e Rio grande do Norte, a utilização desta fibra é bastante intensa no setor de cordaria (cordas, cordéis e tapetes), sendo que pesquisas estão sendo realizadas para a introdução do mesmo como reforço em materiais compósitos, principalmente na área de compósitos poliméricos para a sua utilização na indústria automobilística.

Dessa forma, este trabalho tem como objetivo o desenvolvimento de dois tecidos feitos com fibras de sisal, sendo um deste um tecido plano e outro tecido plano- basket para serem utilizados com reforços para compósitos poliméricos, tendo em vista a inexistência desses na indústria de materiais compósitos. 
Tabela 1. Propriedades Físicas e Mecânicas das Fibras naturais (adaptação de Pardini et al., 2006).

\begin{tabular}{cccc}
\hline $\begin{array}{c}\text { Tipo de } \\
\text { Fibra }\end{array}$ & $\begin{array}{c}\text { Limite de resistência à } \\
\text { Tração (MPa) }\end{array}$ & $\begin{array}{c}\text { Módulo de Elasticidade } \\
\text { (GPa) }\end{array}$ & $\begin{array}{c}\text { Densidade }(\mathbf{g} / \\
\left.\mathbf{c m}^{3}\right)\end{array}$ \\
\hline Sisal & $126-800$ & $3,8-62,0$ & $1,27-1,50$ \\
Coco & $95-149$ & $3,3-5,1$ & $1,18-1,45$ \\
Juta & $320-500$ & $1,3-2,8$ & 1,50 \\
Rami & $392-900$ & $7,3-25,0$ & 1,51 \\
Piaçava & 143 & 5,6 & 1,05 \\
\hline
\end{tabular}

\section{DESENVOLVIMENTO}

Os materiais compósitos são classificados basicamente em dois grupos que são os compostos reforçados por fibras e compostos reforçados por partículas ou compostos particulados (Figura 1). Caracteriza compósitos reforçados com fibras, sendo estas podendo ser continuas, longas, ou curtas, variando o seu comprimento entre $5 \mathrm{~mm}$ e 25 $\mathrm{mm}$ e com relação a sua disposição estas podem ser paralelas, unidirecionais, bidirecionais na forma de tecido, e ainda podem está aleatoriamente orientadas, sendo de fibras contínuas ou picadas (Mendonça 2005).

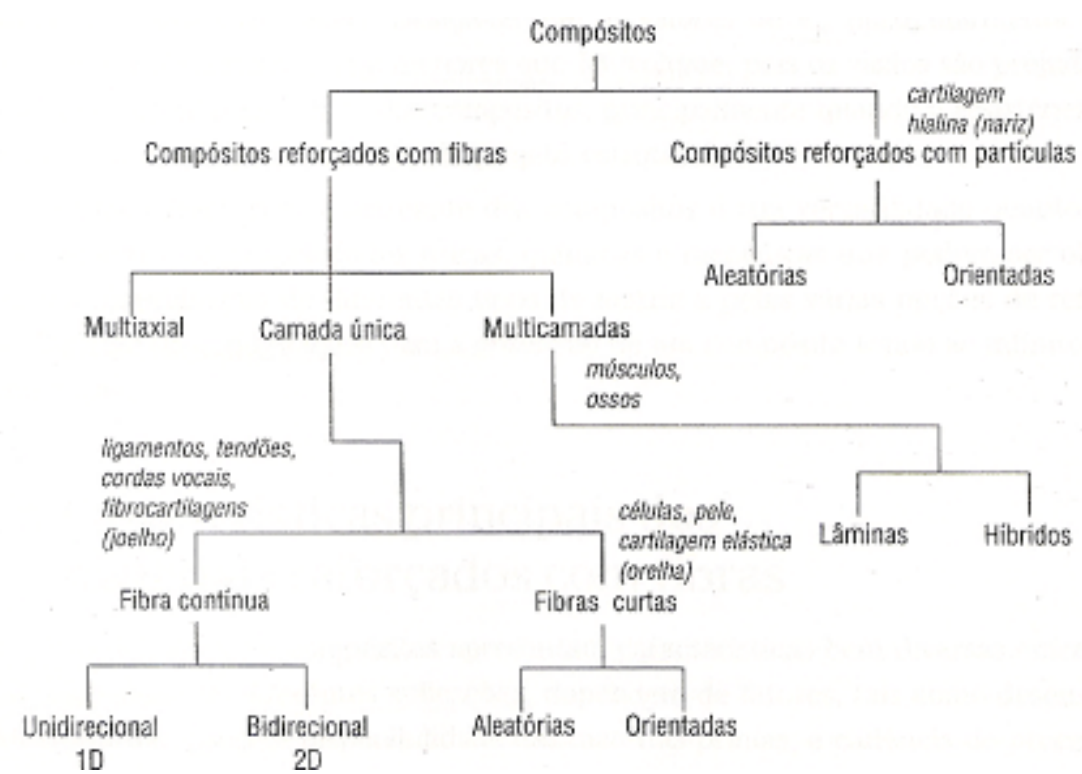

Figura 1. Proposta de Classificação de compósitos sintéticos e naturais.

Os tecidos são fabricados em teares, estes são compostos basicamente por urdume, que dá o comprimento de cada tecido, além disso existe a trama, onde os cabos da trama entrelaçam o urdume, dando a forma e a características do tecido para reforço. A seguir poderão ser observados dois tipos de tecidos diferentes. 


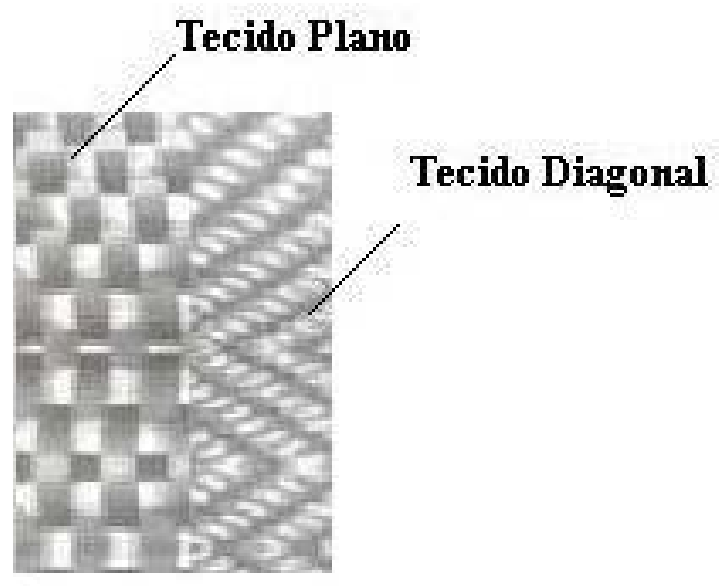

Figura 2. Tipos de tecidos

O tecido plano é caracterizado pelos cabos da trama entrelaçados nos cabos do urdume, onde os cabos da trama se entrelaçam um acima e um abaixo dos cabos do urdume e assim sucessivamente, sendo que na próxima coluna o que estava em cima passa a ser abaixo e 0 que estava abaixo passando por cima e assim sucessivamente ate o final do tecido, como pode ser observado na figura abaixo.

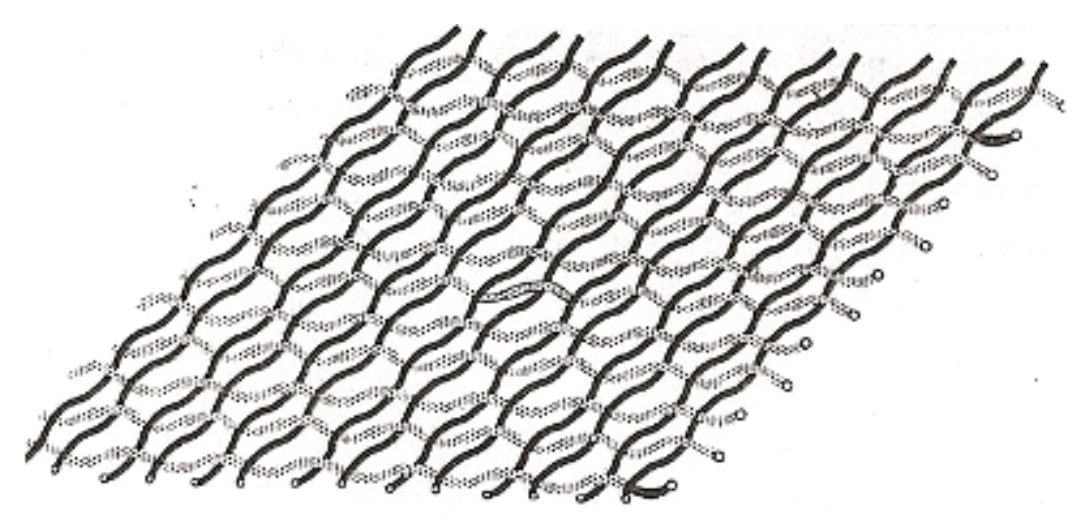

Figura 3. Tecido plano ( Pardine at al., 2006)

Este tipo de tecido possui quatro variações que são os tecidos plano tipo basket $2 x 2$, tecido plano basket do tipo Oxford, tecido Plano tipo Leno e Mock- leno. O tipo plano basket se caracteriza sendo os seus entrelaçamentos urdume, trama da seguinte maneira: dois cabos do urdume se entrelaçam com dois cabos da trama, um acima e um abaixo e assim sucessivamente, já o basket Oxford se caracteriza sendo um cabo da trama se entrelaçando com dois do urdume, dois acima e dois abaixo. Já os tecidos tipos leno os cabos do urdume são entrelaçados a cabos da trama torcidos, formando uma espiral fazendo com que o urdume e a trama fiquem bloqueados a qualquer movimento, e os tecidos planos tipo mock- leno é caracterizado por formarem um entrelaçamento de dois ou mais cabos, e ele possui uma porosidade e uma rugosidade maior do que as outras variações dos tecidos planos (Pardine et all, 2006). 
Os tecidos tipo cetim (harness satin- $H S$ ), são caracterizados por apresentarem um comprimento maior entre as sobrepassagens, seus entrelaçamentos acontecem com no mínimo três cabos acima e um abaixo ( 4 harness satin- $4 H S$ ) e no Maximo de onze cabos acima e um abaixo ( 12 harness satin-12HS), sendo que cada coluna começa um cabo a frente da outra formando uma diagonal no tecido. (Pardine et all, 2006).

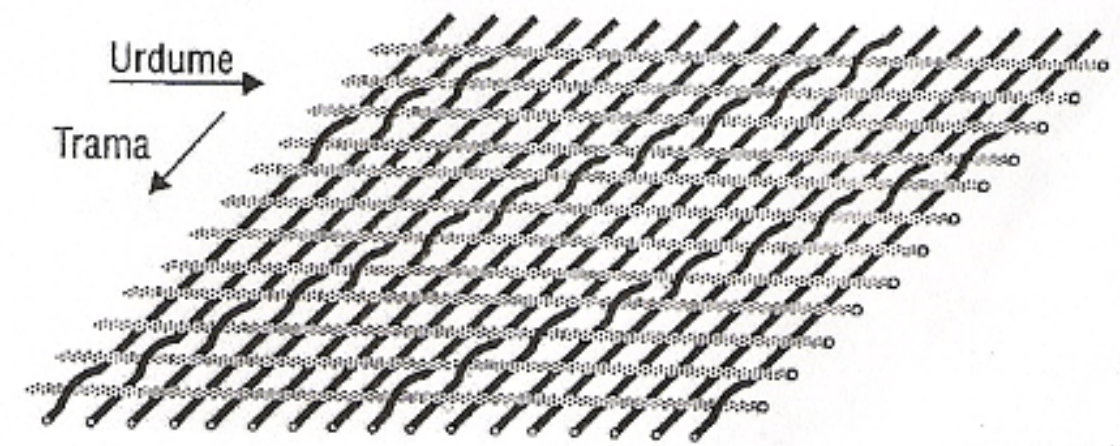

Figura 4. Tecido Cetim ( 8 harnesses - 8 HS), ( Pardine at al., 2006)

O tecido laço Diagonal se caracteriza por apresentar linhas diagonais que formal na parte superior do tecido, os cabos nesse tecido tipo de tecido são colocados dois cabos da trama sobre um cabo o urdume, um abaixo e dois acima, e assim sucessivamente, dessa form formando as diagonais. Esse tipo de tecido possui duas variações o tecido diagonal mão direita que possui as diagonais começando da parte inferior esquerda e chegando a parte superior direita, e o tecido diagonal mão esquerda que começa na parte inferior direita e termina a sua ondulação na parte superior esquerda. ( Pardini et all, 2006).

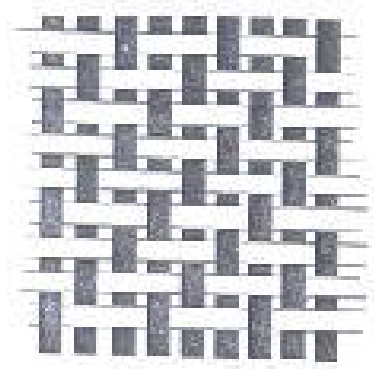

Tecido Mão Direita

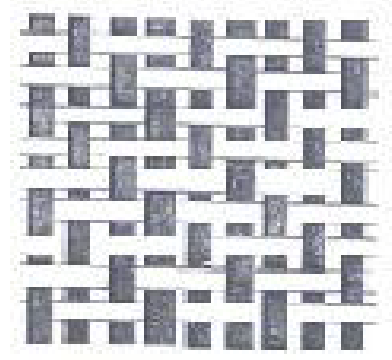

Tecido Mão esquerda

Figura 5. Tecido laço Diagonal ( Pardini et al., 2006)

De acordo com os tipos de tecidos abordados foram escolhidos para este trabalho o tecido plano e sua variação basket 2x2.

\section{MATERIAIS E MÉTODOS}

\section{Materiais}

Foi utilizada para a fabricação do tecido a fibra de sisal com um diâmetro médio de 0,120629 mm e utilizando um tear em aço de 300 x $300 \mathrm{~mm}$. 


\section{Métodos}

Foram confeccionados dois tecidos de sisal, em um tear manual, sendo as configurações dos mesmos; sendo um tecido do tipo plano e o outro do tipo plano basket, porém para todos os tecidos cada cabo possuía 15 fios de sisal, no entanto o tear utilizado media-se $300 \times 300 \mathrm{~mm}$. As fibras foram dispostas no urdume e entrelaçadas pela trama de maneira a obter a primeira etapa de fabricação como demonstra as figuras abaixo:

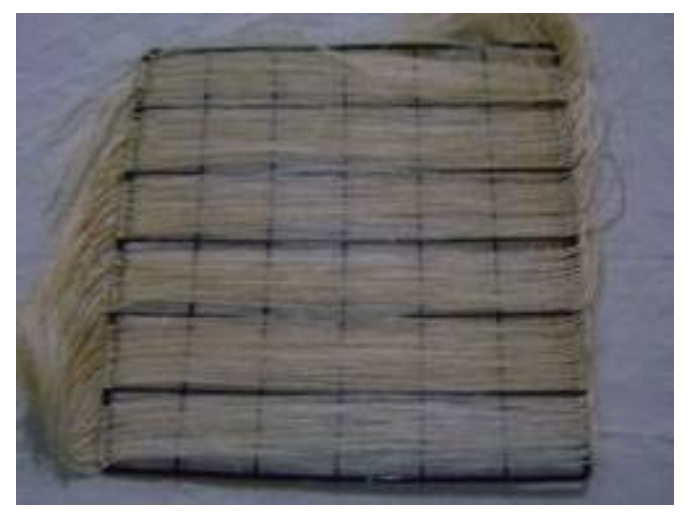

Figura 6. Fibras dispostas na direção do urdume

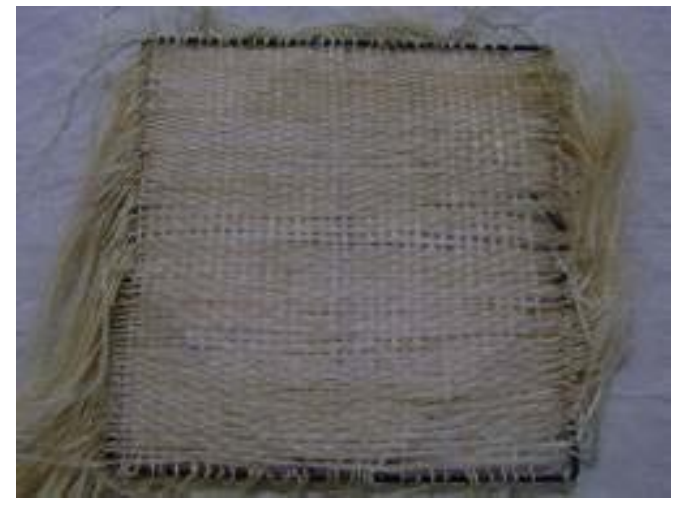

Figura 7. Fibras dispostas no urdume e na trama

\section{RESULTADOS}

A seguir serão apresentados os tecidos que foram fabricados mediante o tear manual. A próxima figura poderá ser observada o tecido plano tipo basket e a seguinte o tecido plano. Para todas as situações a escala utilizada foi em milímetro; verifica-se ainda, que apesar de se utilizar todos os cabos com 15 fios, ao término os tecidos ficaram com gramaturas diferentes, sendo a gramatura do tipo plano basket $440 \mathrm{~g} / \mathrm{m}^{2}$ e do plano $344 \mathrm{~g} / \mathrm{m}^{2}$. Este fato se deu devido à configuração do tear utilizado, desta forma, é interessante que se aprimore este tipo de tear utilizado neste trabalho. 


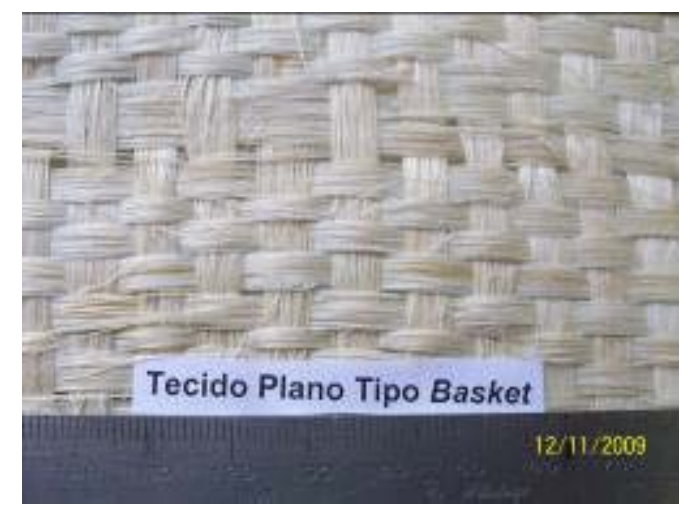

Figura 8. Tecido plano tipo basket

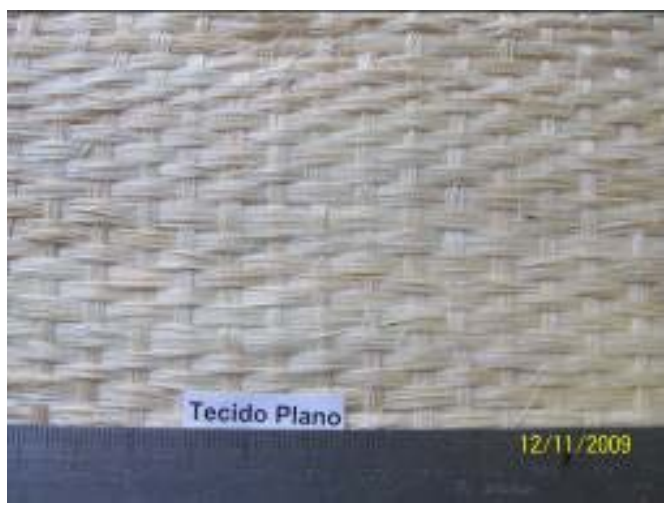

Figura 9. Tecido plano

\section{CONCLUSÕES}

De acordo com o trabalho realizado, vimos que é possível a fabricação de tecidos de sisal para a utilização do mesmo com reforço para materiais compósitos, já que este é inexistente nessa indústria, e com isso além de viabilizar a fibra, estaremos introduzindo emprego ao homem do campo, diminuindo assim o êxodo rural, nas regiões produtoras da fibra de sisal. No entanto como continuidade deste trabalho será a fabricação de um compósito utilizando estes tecidos e determinação do desempenho mecânico destes materiais.

\section{AGRADECIMENTOS}

Agradecemos ao IFRN pela bolsa PIBIC concedida a aluna. 


\section{REFERÊNCIAS BIBLIOGRÁFICAS}

Albuquerque, J. A. C. - O Plástico na Pratica: Manual de Aplicações - Porto Alegre: $2^{a}$ ed.; Editora Sagra Luzzato, 1999.

MENDONÇA, P. T. R. - Materiais Compósitos \& Estruturas-Sanduíches - Barueri, SP: Manoele, 2005.

GAY, D. Matèriaux Composites. Paris: Editions Hermès, 1991.

PARDINI, L. C- Compósitos Estruturais: Ciência e Tecnologia - São Paulo, SP: Edgard Blücher, 2006.

PIGATTO, C.; AMICO. S.C.; MÄHLMANN, C. M.;RODRIGUEZ, A. L - Proprieades Mecânicas e Morfológicas de Blendas de PP/EPDM reforçada com Fibras de Sisal $18^{\circ}$ CBECiMat - Congresso Brasileiro de Engenharia e Ciência dos Materiais, 2008, Porto de Galinhas, PE, Brasil.

ORNAGHI Jr, H. L.; CHIES, D.; FREIRE, E.; AMICO, S. C.; ZATTERA, A. J.Preparação e Caracterização de Compósitos Híbridos Sisal/ Fibra de vidro Processados por RTM.- $18^{\circ}$ CBECiMat - Congresso Brasileiro de Engenharia e Ciência dos Materiais, 2008, Porto de Galinhas, PE, Brasil.

SILVA, R.V. (2003). Compósito de resina poliuretano derivada de óleo de mamona e fibras vegetais. 157 p. Tese (Doutorado) - Instituto de Ciência e Engenharia de Materiais de São Carlos, Universidade de São Paulo, São Carlos, 2003. Brasil.

BARROS A.R de F. Compósitos híbridos: desenvolvimento de configuração e efeito de umificação. 